\title{
Use of the Tangent Chart for Solving Transmission Line Problems
}

\author{
BY RAYMOND S. BROWN \\ Columbus, Ind.
}

\section{INTRODUCTION}

$\mathrm{T}^{\mathrm{m}}$ He general problem of finding the steady-state electrical conditions at some point of a transmission line when they are known at some other point has been a fascinating one for writers, and many schemes, most of them giving approximate solutions, have been proposed for lessening the labor of solving such problems. Dr. A. E. Kennelly has shown that the exact solution of such problems is most simply given by the use of hyperbolic functions and he has been a pioneer in the application of these functions to transmission line problems. About five years ago the writer devised a method, based on hyperbolic functions, for obtaining the exact solution, by applying them in such a manner that the use of tables is unnecessary, provided a special diagram, called a tangent chart, is available. The chart is entirely general in its application, not depending on any particular frequency or length of line. It was described in an article in French, prepared by Messrs. Marius Latour and Geo. Viard from notes furnished them by Dr. C. O. Mailloux, which was published in La Revue Générale de l'Electricité, July 20, 1918. The very favorable consideration which the method received in Europe, and the demand for the publication of a description of the method in the English language, led the writer to take up its study anew, recently, with the idea of improving it, and extending its applicability and practical usefulness. The result is the present paper, which contains an entirely new presentation of the method, in its latest and most complete form.

\section{General Considerations}

In any system of conductors for the transmission of electric power, the dielectric plays just as important a role as does the conductor itself. The dielectric, or shunt, constants, conductance and electrostatic capacity, are always present, even though their effects may be insignificant when compared with those of the series constants, resistance and inductance. It is only when the shunt effects 'are intensified by high voltage, high frequency, or the use of some dielectric other than air, that we must take the shunt constants into account in the solution of transmission line problems. If a line possessed series constants only, or shunt constants only, the fact that these constants were uniformly distributed over the length of line, would have no significance, and problems involving such a line could be solved by the simple application of Ohm's law. Where, however, both kinds of constants are taken into account, their distributed nature must be recognized to get the true solution. Ohm's law can then be applied to a differential length of line, only. The result is, that the expressions for current and voltage, as obtained by integration, are hyperbolic functions of the length of line. In the case of alternating-current transmission, all quantities involved are complex numbers or vectors. In the case of power lines, whose length embraces only a small part of the length of a wave, approximate solutions are frequently used, based upon the expansion of these functions into series. Such approximations do not require the use of tables and give results quite close enough for many purposes provided harmonic frequencies do not need to be taken into account. In the case of telephone lines the length of line embraces an appreciable part of the length of a wave, due to the higher frequency, and approximate solutions are practically never allowable.

In place of the algebraic solution, the writer proposes the use of a chart which will show graphically the electrical conditions at any cross-section of a transmission line operating in the steady state. This graphical method applies to lines having uniformly distributed shunt and series constants and embraces both power and telephone lines of any length, voltage, frequency, or type of construction.

The solution by chart is both simpler and quicker than the algebraic solution, even where approximate equations are used, and it offers the additional advantage that it presents to the eye a picture of the exact electrical condition at every point of the line. It lessens the chance for numerical error and aids greatly in studying the operation of a line, as the effect produced by a change in load or line constants is shown almost at a glance, without the necessity for working through a lot of tedious mathematics. One of the greatest advantages, however, of this method is that is enables the quantity, watts, or effective power at any point of the line, to be represented geometrically by the location of a point in a plane, so that the power may be read directly, without reference to current or voltage, by the mere projection. of this point onto a suitable scale.

In order to understand the theory of this chart a working knowledge of the hyperbolic functions of complex numbers is essential. However, just as a slide rule may be used effectively by one who does not understand logarithms, this chart may be used by those not familiar with hyperbolic functions after they understand the method of procedure. The chart is, in fact, a transmission line slide rule, and its use is similar in many respects to that of the ordinary slide rule. Since it deals with complex numbers in place of scalars it is necessary, however, to construct it in two dimensions in place of one. 


\section{VECTOR EXPRESSION}

There are two methods in general use for expressing the numerical value of a vector or general number; the rectangular form such as $4+j 3$, where $j=\sqrt{-1}$, and the polar form, $5 \sigma^{\mathrm{j} 36.8^{\circ}}$ or, as it is more conveniently written, $5 / 36.8^{\circ}$. In problems involving sums and differences, only, the rectangular form is more convenient to handle, and in problems involving products and ratios only, the polar form is preferable. The method of solution presented here involves products and ratios almost exclusively, so all quantities are expressed in the polar form.

\section{LiNE CONSTANTS}

Let $l=$ the length of the transmission line in miles. $z=r+j x=$ the impedance per mile of conductor. $y=g+j b=$ the admittance per mile of dielectric.

$I$ and $E$ the vector current and voltage respectively, measured to the neutral plane.

$N=\sqrt{z / y}$, a constant usually called the natural impedance, surge impedance, or characteristic impedance of the line. It is defined as the sending end impedance of an infinite length of transmission line having the same cross-section as the actual line. Its dimension is resistance, ohms.

$a=\sqrt{z y}$, is called the propagation constant of the line. Its dimension is $L^{-1}$. The real component of the propagation constant is called the attenuation constant, since it influences the attenuation of current and voltage per mile. The imaginary component is called the wave length constant, since it influences the phase rotation of current and voltage per mile of line.

$A=a l$, is called the complex angle subtended by the line. It is a numeric. The real component is called the attenuation of the line and the imaginary component, the wave length of the line.

The solution of problems relating to the line involves hyperbolic functions of the attenuation, which is thus expressed as a number, and circular functions of the wave length, which may thus be expressed in radians or degrees.

The constants $N$ and $a$ depend upon and replace, in the following analysis, the elementary constants, $z$ and $y$, which are used in approximate solutions. The elementary constants can usually be found tabulated in electrical handbooks. The constants $N$ and $a$, so far as the writer knows, have never been tabulated and must be computed from $z$ and $y$. A table of $N$ and $a$ for various sizes and spacings of open wire would be a valuable addition to the modern handbook as these are the constants used in all exact solutions of transmission line problems.

Fundamental EQUATIONS

The differential equations applying to an element $d l$ of a transmission line, $l$ being measured from the receiving end, are,

$$
\frac{d E}{d l}=z I \quad \frac{d I}{d l}=y E
$$

The solution of these equations gives,

$$
\begin{aligned}
& E=E_{o} \cosh A+N I_{o} \sinh A \\
& I=I_{o} \cosh A+E_{o} / N \sinh A
\end{aligned}
$$

The subscript zero denoting receiving end quantities.

These are the equations ordinarily used for the exact solution. Since they contain both sums and products of vectors they are not well adapted to computation using either the polar or the rectangular form of vector expression. A much more convenient form and one well adapted to solution by chart is obtained as follows:

$$
\text { Let, } \begin{aligned}
W & =\tanh ^{-1} E / N I \\
W_{o} & =\tanh ^{-1} E_{o} / N I_{o}
\end{aligned}
$$

Then,

$$
\begin{gathered}
E / E_{o}=\cosh A+\operatorname{coth} W_{o} \sinh A \\
I / I_{o}=\cosh A+\tanh W_{o} \sinh A \\
\frac{E}{E_{o}}=\frac{\sinh \left(W_{o}+A\right)}{\sinh W_{o}} \\
\frac{I}{I_{o}}=\frac{\cosh \left(W_{o}+A\right)}{\cosh W_{o}}
\end{gathered}
$$

Let,

$$
W=W_{o}+A
$$

Whence,

$$
\begin{aligned}
& E \propto \sinh W \\
& I \propto \cosh W
\end{aligned}
$$

Voltage and current at any point of a transmission line are thus shown to be proportional, respectively, to the hyperbolic sine and cosine of a complex angle, $W$, whose hyperbolic tangent is the ratio of total impedance to natural impedance. Furthermore, the angle $W$ increases uniformly by the amount $a$ for every mile of line passed over. ${ }^{1}$

These equations are well adapted to the polar form of expression, the vector addition $W=W_{o}+A$ being performed graphically by the use of the chart.

\section{The TANGent Graph}

The terminus of the complex angle $W$ may be located in a plane corresponding to any known value of the ratio $E / N I$. The simplest manner in which to locate this point is to construct a graph or Argand diagram of the function, $\tanh W=\tanh (u+j v)$. Such a chart is shown in Fig. 1.

$$
\text { Let } \quad \tanh W=E / N I=T=t / \underline{\phi}
$$

$t$ will represent the magnitude or modulus of $T$ and $\phi$ will represent its direction or argument.

The curves marked with angles in Fig. 1 were plotted by assigning successive constant values to $\phi$ while $t$ was varied, and the curves marked with numbers were plotted by assigning successive constant values to $t$ while $\phi$ was varied. The scalar equations from which these two families of curves were plotted were obtained as follows.

1. The fact that $E$ and $I$ may be expressed as simple hyperbolic sine and consine functions of a complex angle is shown by Dr. A. E. Kennelly in his book "Hyperbolic Functions Applied to Electrical Engineering." 
From the vector equation,

$t(\cos \phi+j \sin \phi)=\tanh (u+j v)$

$$
=\frac{\tanh u+j \tan v}{1+j \tanh u \tan v}
$$

It will be noted that the two families are orthogonal, which tends to make easy the location of points at their intersection.

The $\tanh W$ function is periodic in the direction of the $V$ axis, repeating in the interval $j \pi$. It is non-

Graph of TaNH $(u+j v)$

s Scale

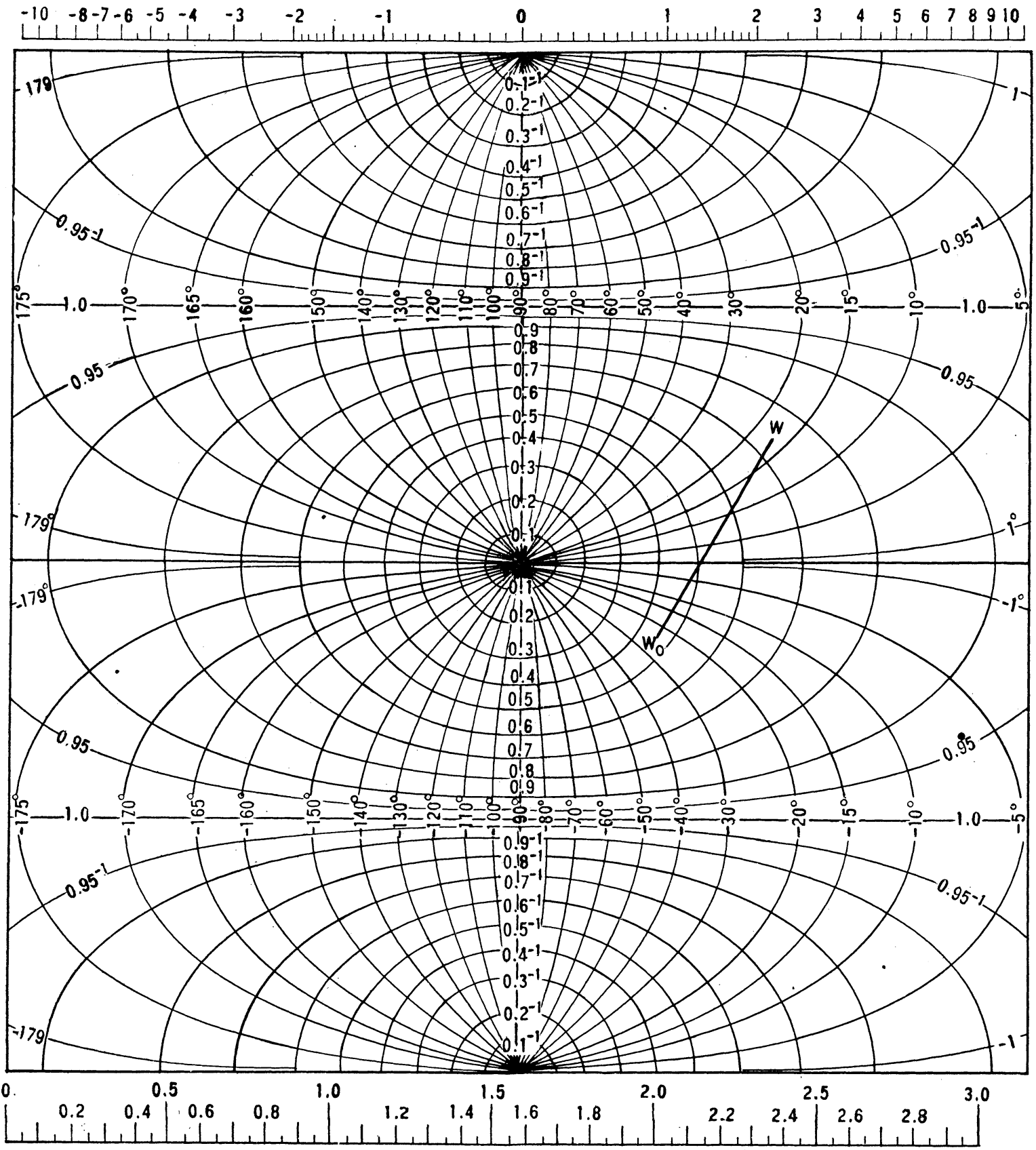

A SCALE

FIg. 1

we get the two scalar equations,

$$
\begin{aligned}
& \frac{\sin 2 v}{\sinh 2 u}=\tan \phi \\
& \frac{\cos 2 v}{\cosh 2 u}=\frac{1-t^{2}}{1+t^{2}}
\end{aligned}
$$

as the equations of these curves. periodic in the direction of the $U$ axis. The graph may thus be thought of as the surface of a cylinder, of diameter equal to unity, which for convenience has been cut parallel to the $U$ axis and spread out into a plane.

Since the circular and hyperbolic tangents bear the relation $\tan W=-j \tanh j W$, the graph of $\tanh W$, Fig. 1, becomes also the graph of $\tan W$ if the chart is rotated $90 \mathrm{deg}$. and the numbers on each of the angle 
curves reduced by 90 deg. The $\tan W$ function is thus periodic in the direction of the real axis and nonperiodic in the direction of the imaginary axis.

If we compute the numerical value of the ratio $E / N I$ in the polar form we can readily locate the point $W$ by the use of this chart. Supposing known conditions to be at the receiving end of the line, we designate the corresponding value of $W$ by $W_{o}$. If we add graphically to $W_{\circ}$ the vector $A=a l$, by laying it off from the point $W_{0}$ we arrive at the point $W$ since by equation $5, W=W_{o}+A$. The value of $t / \phi$ read from the curves at the point $W$ gives us the ratio $E / N I$ at the sending end of the line. The angle $A$ being directly proportional to length of transmission line, enables us, should we so desire, easily to find the ratio $T$, at any intermediate point on the transmission line. Multiplying $T$ by $N$ gives us the sending end impedance $E / I$ at the point $W$.

Should the point $W$ fall at the center of Fig. 1 it indicates the condition $E / N I=0$ or the corresponding point on the transmission line is grounded, $E=0$. Should $W$ fall at the other focus of the chart it indicates the condition $N I / E=0$ or the corresponding point on the transmission line is free, $I=0$.

The region beyond the right and left hand boundaries of Fig. 1 indicates $T=1$ or the sending end impedance at every point is equal to the natural impedance. This condition exists when the load is of such a character that waves of current and voltage sent out by the generator suffer no terminal reflection. When $W$ travels in this region the phase relation between $I$ and $E$ remains constant and, as $W$ recedes from the $V$ axis both $I$ and $E$ increase in the ratio, $\sigma^{a}$ for every mile of line passed over.

The tangent chart illustrates very well the so called Ferranti effect, such as is exhibited by long power lines when operating under light load. In this condition the line acts somewhat like a transformer and converts from low voltage and large current at the sending end to high voltage and small current at the receiving end. An inspection of Fig. 1 shows this effect to be most pronounced when the length of transmission line is a quarter wave length, $A=j 1 / 2 \pi$, as $A$ is then just long enough to span from the $E=0$ focus to the $I=0$ focus. The condition $A=j 1 / 2 \pi$ is never fully attained in practise due to dissipation of power in the conductor and dielectric, consequently the vector $A$ always has a real component.

\section{Voltage AND CuRrent}

We have seen that the tangent chart enables us to find the value of total impedance at any point of the transmission line. We shall now show how voltage and current may be obtained at this point.

Let, $H=\sinh 2 W=2 \sinh W \cosh W$

Whence, $H \propto E I$ and, since $T \propto E / I$

$$
E \propto \sqrt{H T} \quad I \propto \sqrt{H / T}
$$

$H$ is given in terms of $T$ by the hyperbolic relation,

$$
H=\frac{2 T}{1-T^{2}}
$$

Having found from the chart the values of $T$ at any two points on the transmission line, and knowing the values of $E$ and $I$ at one of these points, we can thus find, by simple proportion, the values of $E$ and $I$ at the other point. $E$ and $I$ thus determined are vector quantities, the phase angles of which are measured with respect to the voltage (or current) at some arbitrary point, for instance, the receiving end of the line.

In the great majority of problems we are not interested in the phase of $E$ and $I$, individually, but only in their relative phase, as it is the angle included between these two vectors which determines the power factor. This relative phase is fully determined by the ratio, $T$, as found from the chart. We are, however, interested in the individual scalar values of $E$ and $I$. It is possible to find these scalar values much more easily than by the use of the above equation for $H$, as this equation involves both sums and ratios of vectors and is, therefore, not well adapted to computation.

Let, $e=$ the scalar value of $E$

$i=$ the scalar value of $I$

$h=$ the scalar value of $H$

Then,

$$
\begin{aligned}
& e \propto \sqrt{h t} \quad i \propto \sqrt{h / t} \\
& \frac{1}{2 H}=\frac{1}{T}-T=\frac{1}{t}(\cos \phi-j \sin \phi) \\
& \quad-t(\cos \phi+j \sin \phi) \\
& \quad=\frac{1-\cos ^{2} \phi}{\sqrt{\left.1 / 4(t+1 / t)^{2}-10\right)}}
\end{aligned}
$$

We can then find the scalar values of $E$ and $I$ at any point on the line by means of the proportions,

$$
\begin{aligned}
\frac{e}{e_{o}} & =\sqrt{\frac{h t}{h_{o} t_{o}}} \\
\frac{i}{i_{o}} & =\sqrt{\frac{h t_{o}}{h_{o} t}}
\end{aligned}
$$

These are scalar equations and well adapted to computation.

Should the point $W$ fall at the $E=0$ focus of the chart, we have by equation (10)

$$
\begin{array}{llll}
t=0 & h=0 & \sqrt{h t}=0 & \sqrt{h / t}=\sqrt{2}
\end{array}
$$

and should it fall at the $I=0$ focus we have,

$$
1 / t=0 \quad h=0 \quad \sqrt{h} t=\sqrt{2} \quad \sqrt{h / t}=0
$$

In place of obtaining $h$ from equation 10 it may be found more easily by expressing it in terms of $u$ and $\phi$ thus,

$$
H=\sinh 2 W=\sinh 2 u \cos 2 v+j \cosh 2 u \sin 2 v
$$$$
h=\sinh ^{2} 2 u+\sin ^{2} 2 v
$$

Eliminating $v$ by means of equation 8 ,

$$
h=\frac{\sinh 2 u}{\cos \phi}=\stackrel{s}{\cos \bar{\phi}}
$$


The use of this equation requires an additional scale, that of $s=\sinh 2 u$, which is shown at the top of the chart. This scale also serves another useful purpose as will be shown later. It is read by projecting vertically from the point $W .^{2}$

Equation 13 becomes indeterminate for all points on the $V$ axis, $u=0, \phi= \pm 90$ deg., and equation 10 must be used in its stead for such points.

\section{POWER RELATIONS}

If $\delta$ represents the argument of vector $N$ and $\beta$ the argument of vector $I, E$ being taken as standard phase or zero argument,

$$
t / \phi=E / N I \quad \phi=-\delta-\beta
$$

Let $p$ be the effective power or watts, then,

$$
\begin{aligned}
p \propto h \cos \beta & =\frac{\sinh 2 u}{\cos \phi} \cos (\phi+\delta) \\
& =\sinh 2 u(1-\tan \delta \tan \phi) \cos \delta
\end{aligned}
$$

$\cos \delta$ being a constant, may be dropped from the proportion. Eliminating $\phi$ by means of equation 8 ,

$$
p \propto \sinh 2 u-\tan \delta \sin 2 v
$$

The power will be zero when

$$
\frac{\sin 2 v}{\sinh 2 u}=\cot \delta=\tan ( \pm \pi / 2-\delta)
$$

This is the equation of that particular angle curve on the chart, Fig. 1, for which $\phi= \pm \pi / 2-\delta$ or $\beta= \pm \pi / 2$

But $\beta= \pm \pi / 2$ implies that current and voltage are in quadrature. Hence all points on this particular angle curve represent the condition of zero power factor and this curve may thus be designated the zero power factor wave.

This wave makes the angle $\delta$ with the $V$ axis at the origin. Similarly we have a unity power factor wave $\frac{\sin 2 v}{\sinh 2 u}=\tan -\delta$ making the angle $\delta$ with the $U$ axis at the origin.

Denoting the abscissa of the zero power factor wave by $u^{\prime}$ we get,

$$
\begin{aligned}
& p \propto \sinh 2 u-\sinh 2 u^{\prime} \\
& p \propto s-s^{\prime}
\end{aligned}
$$

The result of this analysis is shown graphically in

2. If it were possible, without confusion of lines, to superpose on the tangent chart a graph of $\sinh W$ or $\cosh W$, in which modulus curves only, were shown, each curve being marked with the parameter used in plotting it, we should have a means of finding the scalar values of $E$ and $I$ without the use of the $s$ scale.

This follows from equations 6 and 7 .

The tangent chart, as originally published, under the name of "Transmission Line Coordinate Paper," used this method, the modulus curves being drawn on the back of the chart and read by holding the chart up to transmitted light. It is believed that the present method is simpler, and in addition it permits the chart to be reproduced on an opaque surface.

The article by Messrs. Latour and Viard referred to in the introduction, contains a good description of the tangent chart, its mathematical basis and derivation and the practical use of the original form of tangent chart devised by the writer.
Fig. 2 where the zero power factor wave is shown for one and a half cycles. In this particular case $\delta$ is a negative angle.

The inclined straight line represents the locus of the point $W$. This line, if sufficiently extended, will cut the zero power factor curve in one point and only one. This corresponds to a point on the transmission line at which the power factor is zero but neither current nor voltage is zero. In other words, power is flowing toward this point from both directions, as would be the case with a transmission line fed with power from

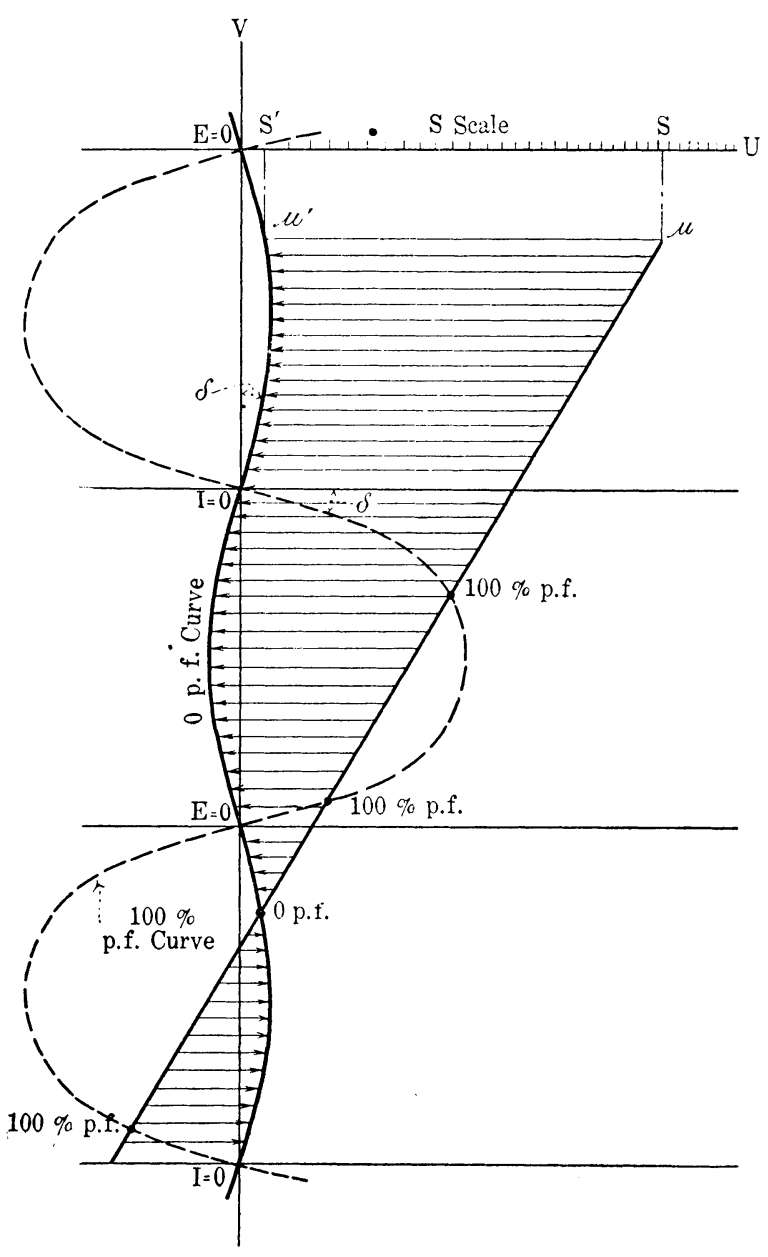

FIG. 2

two generating stations. The dotted line represents the locus of the unity power factor wave, $\phi=-\delta$. This curve will, in general, be intersected in more than one point, each intersection corresponding to the condition of unity power factor on the transmission line.

Since $\sinh 2 u$ always increases as $u$ increases, the intercepted horizontal distance, shown shaded on the chart, may be considered as a rough measure of the power at any point.

The exact watt efficiency of any transmission line is given by,

$$
f=\frac{s_{o}-s_{o}{ }^{\prime}}{s-s^{\prime}}
$$

When $N, A$, and the load impedance are known the 
efficiency of any line may thus be very simply found, entirely without reference to current or voltage.

The maximum possible variation of $\delta$ is from $-45 \mathrm{deg}$. to +45 deg. Thus $s^{\prime}$ can never be greater than unity, as represented by the projection of the crest of the wave.

Power flow is represented on the chart as always being toward the zero power factor wave. The receiving end of any $A$ line which may be drawn on the chart will thus be whichever end of the $A$ line is nearest this curve. Since $\phi$ usually lies between $-90 \mathrm{deg}$. and +90 deg. the point $W$ usually falls on the right side of the $V$ axis and the left end of the $A$ line is thus ordinarily the receiving end.

\section{MAXIMUM EFFICIENCY}

We have seen that for every position of the $A$ line in the plane of the chart the corresponding watt efficiency of the transmission line may be readily found by the use of the zero power factor wave and the $s$ scale. As the position of the $A$ line recedes from the $V$ axis the imaginary component of $A$, or the wave length of the transmission line, has less and less effect on the efficiency, until, when $A$ reaches the region where $T=1$, the efficiency approaches the constant value,

$$
f=\frac{\sinh 2(u+c)}{\sinh 2 u} u=\infty \sigma^{2 c},
$$

$c$ being the attenuation. The efficiency, $\sigma^{2 c}$, is however, the maximum efficiency only in the special case where $\delta=0$, or the ratio $x / r=b / g$. This of course, includes the case of direct current where both $x$ and $b$ are zero. In all other cases the perturbing effect of the zero power factor wave is such as to produce an efficiency somewhat higher than $\sigma^{2 c}$ at a certain location in the plane. In order to find this particular location it is necessary to vary the position of $A$ with respect to the $U$ and $V$ axes independently and solve the resulting equations simultaneously. This is accomplished as follows.

The efficiency pertaining to any location of the $A$ line is,

$$
f=\frac{\sinh 2 u_{o}-\tan \delta \sin 2 v_{o}}{\sinh 2 u-\tan \delta \sin 2 v}
$$

$u, v$ being the coordinates of the sending end and $u_{o}, v_{o}$ of the receiving end.

Since $f$ is to be a maximum with respect to both $u$ and $v$,

$$
\frac{\partial f}{\partial u}=0 \quad \text { and } \quad \frac{\partial f}{\partial v}=0
$$

whence,

$(\sinh 2 u-\tan \delta \sin 2 v) \cosh 2 u_{o} \partial u_{o}=$

$\left(\sinh 2 u_{o}-\tan \delta \sin 2 v_{o}\right) \cosh 2 u \partial u$

$(\sinh 2 u-\tan \delta \sin 2 v) \cos 2 v_{0} \partial v_{0}=$

$\left(\sinh 2 u_{o}-\tan \delta \sin 2 v_{o}\right) \cos 2 v \partial v$

But, since $u$ and $u_{o}$ differ by a constant and $v$ and $v_{0}$ differ by a constant, $\partial u_{o}=\partial u$ and $\partial v_{o}=\partial v$

Whence, $f=\frac{\cosh 2 u_{o}}{\cosh 2 u}$ and $f=\frac{\cos 2 v_{o}}{\cos 2 v}$
Hence, $\frac{\cos 2 v_{o}}{\cosh 2 u_{o}}=\frac{\cos 2 v}{\cosh 2 u}$

$$
\frac{1-t_{o}^{2}}{1+t_{o}^{2}}=\frac{1-t^{2}}{1+t^{2}} \text { or } t_{o}=t
$$

Also, $\quad \frac{\sinh 2 u_{o}-\tan \delta \sin 2 v_{o}}{\sinh 2 u-\tan \delta \sin 2 v}=\frac{\cosh 2 u_{o}}{\cosh 2 u}$

Replacing $u$ and $v$ by $\phi$ and $t$, by the use of equation 8 and 9 and using the relation found above, $t_{o}=t$,

$$
\frac{1-\tan \delta \tan \phi_{o}}{1-\tan \delta \tan \phi}=\frac{\cos \phi}{\cos \phi_{0}}
$$

$\cos \delta \cos \phi_{o}-\sin \delta \sin \phi_{o}=\cos \phi-\sin \delta \sin \phi$

$$
\begin{aligned}
\cos \left(\delta+\phi_{o}\right) & =\cos (\delta+\phi) \\
\delta+\phi_{o} & = \pm(\delta+\phi)
\end{aligned}
$$

Ignoring the plus sign which gives $W=W_{0}$ we have,

$$
\begin{aligned}
& \delta+\phi_{o}=-\delta-\phi \\
& \phi_{o}+\phi=-2 \delta \quad \text { or } \quad \beta_{o}=-\beta
\end{aligned}
$$

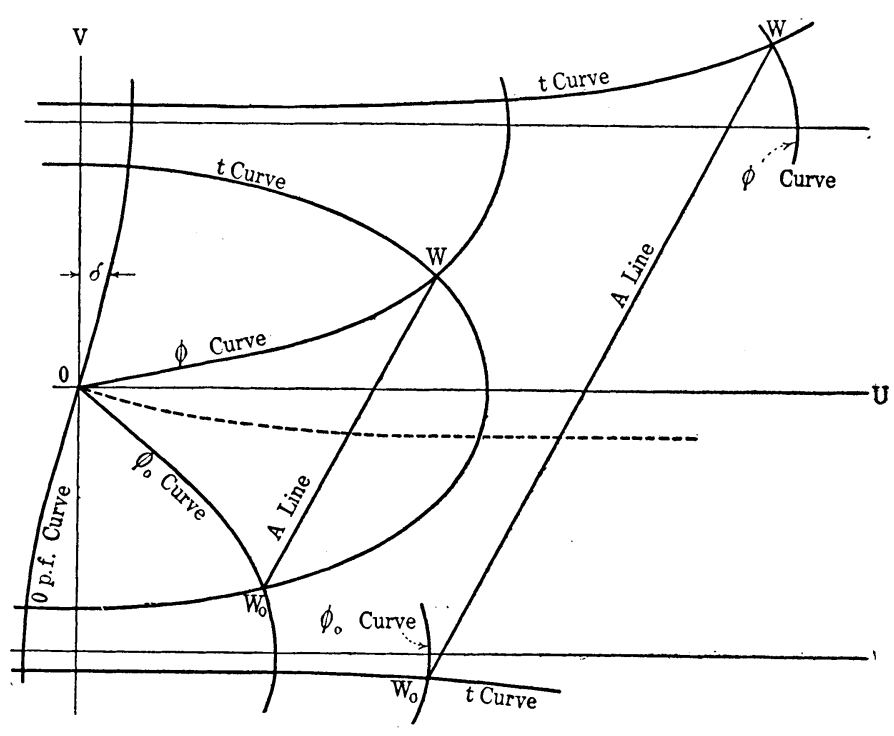

FIG. 3

The result of this analysis may be stated as follows. Any transmission line will operate at its maximum efficiency when the impedances at the opposite ends of the line are symmetrical. That is, the scalar values of the impedances and also the power factors must be equal at the opposite ends of the line but the current must lead the voltage at one end by the same angle that it lags at the other.

The location of the $A$ line on the chart to attain this maximum efficiency may be found by the use of the $\phi, t$ curves and entirely without numerical computation. This is accomplished as follows, see Fig. 3.

Having laid the $A$ line off along the edge of a strip of paper, move it slowly toward the $V$ axis, being careful to preserve the proper slope and to keep the extremities, $W$ and $W_{o}$, of the $A$ line, on the same $t$ curve at any position. The midpoint of the $A$ line will move along a locus represented by the dotted line. Note the algebraic sum of $\phi+\phi_{0}$ as $A$ approaches the $V$ axis. 
When this sum reaches the value $-2 \delta$, the position of maximum efficiency will have been reached.

Since $\delta$ is the argument of the vector $\sqrt{z / y}$,

$$
2 \delta=\tan ^{-1} x / r-\tan ^{-1} b / g
$$

Hence when $b / g$ is greater than $x / r, 2 \delta$ is negative and $\phi+\phi_{0}$ is positive. The point $O$, Fig. 3 , then represents the $I=0$ focus of the chart, Fig. 1 , because in this region $\phi_{o}$ is positive and numerically greater than $\phi$.

When $b / g$ is less than $x / r, \phi+\phi_{\circ}$ is negative and $O$ represents the $E=0$ focus.

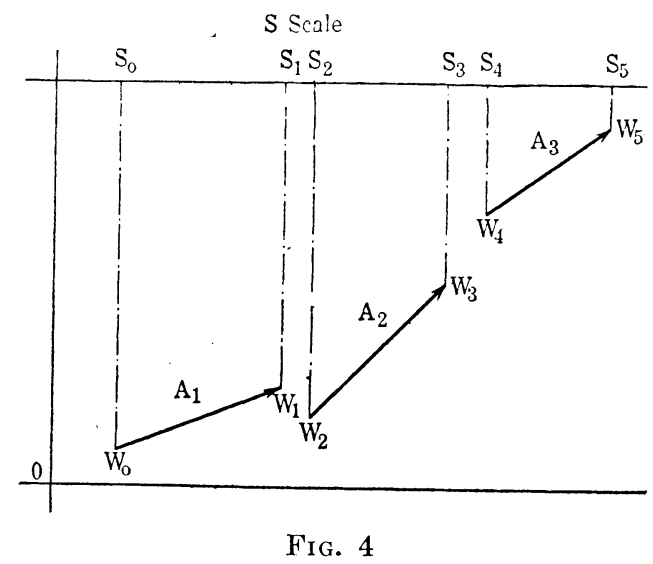

The construction at the right of Fig. 3 shows the location of $A$ when the length of transmission line is greater than a quarter wave length.

In the case of power lines having no leakage, the $A$ line, for maximum efficiency, lies with its mid-point very near to the $I=0$ focus. The load current is laging nearly $90 \mathrm{deg}$. and the sending end current leading nearly $90 \mathrm{deg}$. This condition is only attained in practise on light, highly inductive load, such as transformer charging current. At full load the average power line works at somewhat less than maximum efficiency.

\section{Composite LiNeS}

The tangent chart may be used to good advantage for the solution of problems relating to composite transmission lines, that is to say, lines made up of sections in series having different constants, as for example, open wire and cable.

In general the locus of the point $W$ for such a line will be a series of disconnected straight lines, each being the $A$ line for that particular section, see Fig. 4 . $W_{2}$ is found from $W_{1}$ by means of the relation,

$$
T_{2} / T_{1}=N_{1} / N_{2}
$$

$N_{1}$ and $N_{2}$ being the natural impedances of the sections. $I$ and $E$ are the same on both sides of any junction.

The volt-ampere equation for such a composite line takes the form,

$$
\frac{e_{5} i_{5}}{e_{o} i_{o}}=\frac{h_{1} h_{3} h_{5}}{h_{o} h_{2} h_{1}}
$$

Having thus found $T$ and $e i$ at the distant end of the line, current and voltage are completely determined. The various $A$ lines will alter their relative positions in the plane with change of load. Their individual lengths and slopes will remain constant however.

In the special case where the natural impedances of the various sections of line are all the same, the $W$ locus becomes a continuous, broken line, and the line $A$, Fig. 5 , representing the sum of the sections, may be used in their place. It is interesting to note that the terminal load is equivalent in its effect to an imaginary section of line, of the same natural impedance as that of the sections and whose value of $A$ is $W_{0}$. The distant end of this imaginary section may be conceived as either grounded or free, depending upon whether the point $O$ is the $E=0$ or the $I=0$ focus of the chart.

\section{SerIes LoAdS}

The solution for irregularly loaded telephone lines may readily be found by the use of the chart. The loads may be either series or shunt.

In the case of series loading, the terminal impedance being known, $W_{o}$ is determined, Fig. 4. From this point lay off the vector $A_{1}$, corresponding to the first section of uniform line. This brings us to the first load at which we obtain the sending end impedance $E_{1} / I_{1}$ $=N_{1} T_{1}$ on the receiver side of the load. The impedance on the sending side of the load is $\left(N_{1} T_{1}+Z\right)$ where $Z$ is the impedance of the load. Hence,

$$
T_{2}=\frac{\left(N_{1} T_{1}+Z\right)}{N_{2}}
$$

and the point $W_{2}$ is determined.

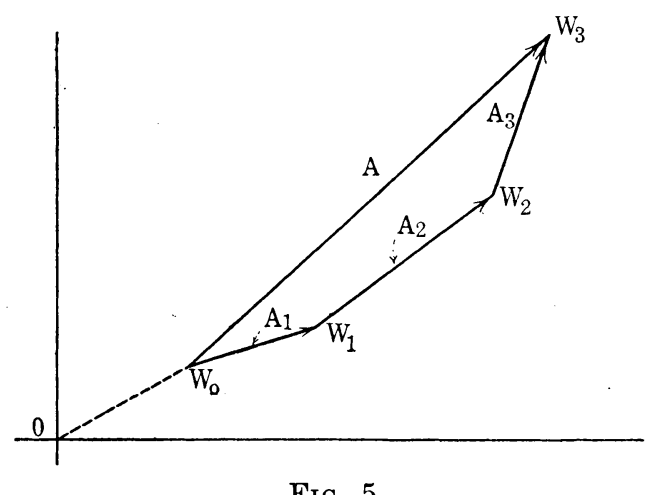

From $W_{2}$ we proceed with the next uniform section of line and so on until all loads and all sections have been passed and we arrive at the final sending end impedance. The addition of impedances may be performed graphically on a separate sheet if desired.

The efficiency of such a loaded line is the product of the efficiencies of all the loads and all the sections. The efficiency of any uniform section of line is given by equation 15. The efficiency of any series load is,

$$
\begin{aligned}
f_{\text {s }}=1-\frac{\text { loss }}{\text { input }}=1- & \frac{r i_{2}^{2}}{e_{2} i_{2}}-\frac{r}{\cos \beta_{2}} \\
& =1-\frac{r}{t_{2} n_{2} \cos \beta_{2}}
\end{aligned}
$$


where $r$ is the resistance of the load, $t_{2}, n_{2}, \beta_{2}$ are measured on the sending side of the load and $n_{2}$ is the scalar value of $N_{2}$.

\section{ShUNT LOADING}

Lines containing shunt loads are solved in a similar manner except that the admittance is found at the sending end of each load and added vectorially to the admittance of the load. The corresponding formula for $T_{2}$ then becomes, $1 / T_{2}=N_{2}\left(1 / N_{1} T_{1}+Y\right), \quad Y$ being the admittance of the load. The formula for efficiency of a load becomes,

$$
\begin{aligned}
f_{p}=1-\frac{\operatorname{loss}}{\text { input }}=1-\frac{g e_{2}^{2}}{e_{2} i_{2} \cos \beta_{2}} \\
=1-\frac{t_{2} n_{2} g}{\cos \beta_{2}}
\end{aligned}
$$

where $g$ is the conductance of the load.

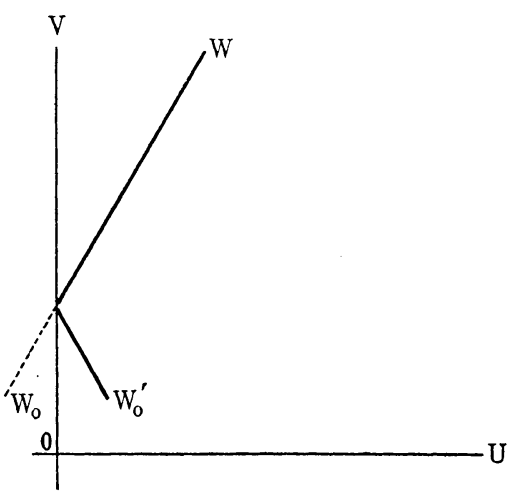

FIG. 6

This same method of solution may be applied to power lines having loads of known admittance tapped off at intervals, except that in this case the efficiency of the loads does not enter as a factor in the total line efficiency.

\section{DiRECT-CURRENT LINES}

In the case of direct-current transmission lines such as cables, having distributed leakage and resistance, the constants $N$ and $A$ become real numbers and the $A$ line lies in the $U$ axis of the chart. The formulas for $h$, and $e$ and $i$ become,

$$
h=s \quad e \propto \sqrt{s t} \quad i \propto \sqrt{s / t}
$$

The similarity in principle between the tangent chart and the ordinary slide rule is quite evident in the case of direct current as both the tangent scale and the $A$ scale consist of real numbers and are capable of being used on slides in the same manner as are the usual logarithmic scales.

\section{The Work CharT}

In using the tangent chart for numerical computation it is desirable, for reasons of accuracy, that it be drawn to as large a scale as possible. An inspection of Fig. 1 shows that, so far as the shape of the curves is concerned, regardless of the numbers attached to them, the diagram is symmetrical about the $V$ axis and repeats itself at intervals of a quarter wave length, $1 / 2 \pi$, along the $V$ axis. This fact is taken advantage of in the construction of the work chart, which is an enlarged reproduction of the right central portion of Fig. 1. The curves are plotted to a scale twice as large as would be possible if the entire function were shown.

On the work chart, values of $T$ greater than unity cannot be plotted. Due, however, to the relation, $\operatorname{coth} W=\tanh (W \pm j 1 / 2 \pi)$

we may invert $T$ whenever it is greater than unity, plot the resulting angle, and carry out the solution just as though we were working on the missing half of the chart. Our final result will then be read as $1 / T$ or $N I / E$, which is the value of $\operatorname{coth} W$.

The left half of the tangent diagram has been omitted from the work chart for the reason that in most practical problems $W$ will lie to the right of the $V$ axis. In case, however, the $A$ line should cross the $V$ axis, it may be shown as reflected from the axis, as in Fig. 6 . The value of $\phi$ as read at $W^{\prime}{ }_{0}$ will then be the supplement of the actual value at $W_{0}$. The value of $t$ is the same at both points.

In case the $A$ line runs off the top or bottom boundary of the chart it reappears at the opposite side and continues in the same direction as before, such that if the chart were rolled into a cylinder, parallel to the $U$ axis, the $A$ line would form a helix, see Fig. 7. In this case the $A$ line is broken into two or more parts, as, $A_{1}, A_{2}, A_{3}$. If points on the line $A_{1}$ read the ratio $E / N I$ then points on $A_{2}$ will read $N I / E$, the ratio, $T$, inverting every time $W$ passes off one boundary and reappears at the other.

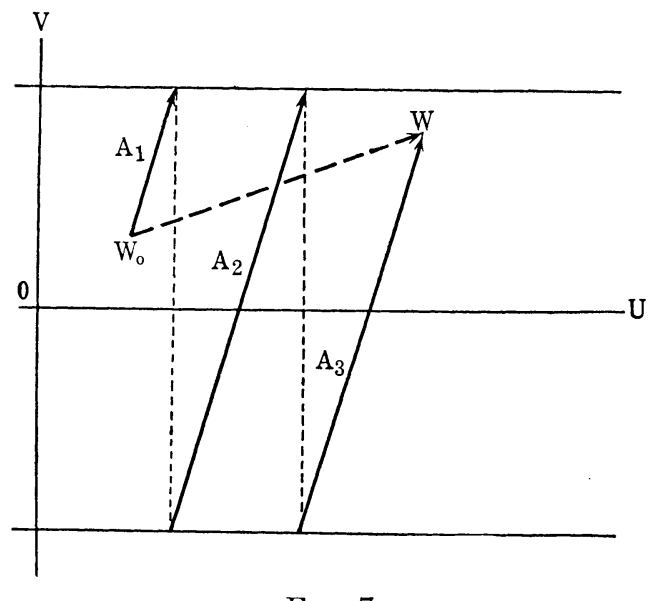

FIg. 7

For very long lines, an effective value of $A_{\epsilon}$, may be substituted for $A$. This is a fictitious value of $A$ which will produce the same terminal effects as the actual value. When this substitution is made care must be taken to note whether $W_{0}$ and $W$ read the same or inverse ratios of $T$.

It will be noted that the curves on the work chart are symmetrical about the $U$ axis. This permits a chart of the top half, only, of the work chart to be used 
if desired. Such a chart would be only one eighth of a wave length in height and would permit even a larger scale to be used, with a corresponding increase in accuracy. The locus of the point $W$ on such a chart would appear as in Fig. 8. The sign of the angle $\phi$ would reverse with every reflection from the bottom boundary and the ratio $T$ would invert with every reflection from the top boundary. The point $W$ would follow the same path as would a billiard ball if rolled on a table, the cushions of which represent the boundaries of the chart.

\section{USE OF THE WORK CHART}

The work chart, as here presented, may be used for the solution of transmission line problems without drawing upon it if a small triangle, representing the vector, $A$, be cut out of cardboard and laid upon the chart with its base parallel to the $U$ axis. It may be

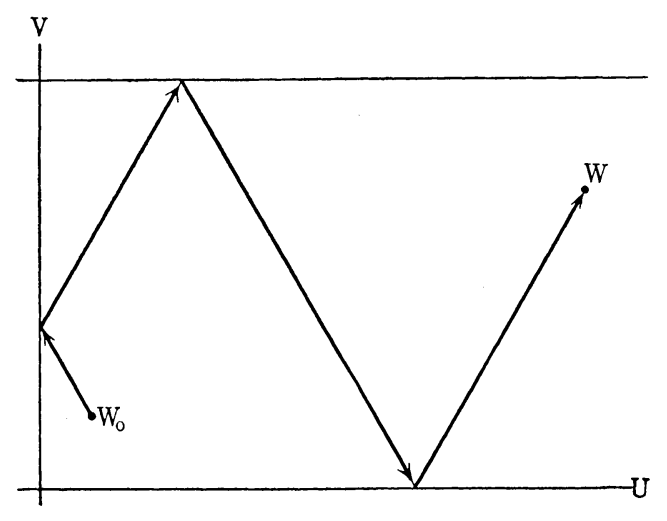

Fig. 8

moved about to represent various electrical conditions on the transmission line and the values of $T_{0}$ and $T$ read off at the corners. In place of using cardboard, $A$ may be drawn on a piece of tracing cloth and moved about over the chart. When using the work chart it is desirable to have it attached to a drawing board so that the $s$ scale may be read by projection. The angle $\alpha$ has a possible variation of 90 deg. each way from zero but in most practical problems will be found to lie between 80 and $90 \mathrm{deg}$.

\section{Numerical EXAMPLE}

A three-phase power line has the following constants per wire,

$$
\begin{aligned}
Z= & 60+j 165=175.5 / 70^{\circ} \\
& \text { ohms } \\
Y= & 0+j 0.0012=0.0012 / 90^{\circ} \\
& \operatorname{mhos} \\
\text { Whence, } \quad N= & \sqrt{Z / Y}=382.5 /-10^{\circ} \\
& \text { ohms } \\
A= & \sqrt{Z Y}=0.459 / 80^{\circ}
\end{aligned}
$$

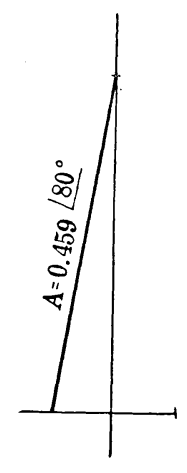

If the voltage to neutral at the load is 60,000 volts and the load current is $90 /-20^{\circ}$ amperes, find the conditions at the sending end.

$$
\begin{aligned}
& \text { SOLUTION } \\
& \frac{1}{T_{o}}=-\frac{N I_{o}}{E_{o}}=\frac{382.5 /-10^{\circ} \times 90 /-20^{\circ}}{60000} \\
& =0.574 /-30^{\circ}
\end{aligned}
$$

$T_{o}$ being greater than unity, the inverse ratio must be used. Plotting the value $1 / T_{o}$ we read $s_{o}=1.115$ whence,

$$
h_{o}=\frac{s_{o}}{\cos \phi_{o}}=\frac{1.115}{\cos -30^{\circ}}=1.288
$$

Laying off vector $A$ from the point $W_{o}$, we reach $W$, from which we read the conditions at the sending end,

$$
\begin{aligned}
& \frac{1}{T}=\frac{N I}{E}=.516 / 8^{\circ} \\
& h=\frac{s}{\cos \phi}=\frac{1.36}{\cos 8^{\circ}}=1.373
\end{aligned}
$$

Hence the phase of sending end current with respect to sending end voltage is $\beta=8-\delta=18^{\circ}$ lead.

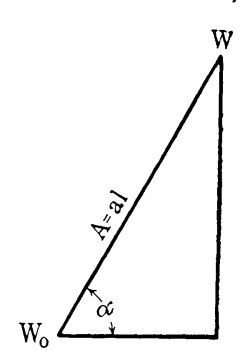

FIg. 9
The scalar values of sending end voltage and current are found by the use of equations 11 and $\mathbf{1 2}$ to be,

$e=e_{o} \sqrt{\frac{h t}{h_{o} t_{o}}}=60,000 \sqrt{\frac{1.373 \times 0.574}{1.288 \times 0.516}}$

$i=i_{o} \sqrt{\frac{h t_{o}}{h_{o} t}}=90 \sqrt{\frac{1.373 \times 0.516}{1.288 \times 0.574}}$
$=88.2$ amperes, scalar value.

The efficiency of transmission is,

$f=-\frac{h_{o} \cos \beta_{o}}{h \cos \beta}=\frac{1.288 \times \cos -20^{\circ}}{1.373 \times \cos 18^{\circ}}$

$=92.8$ per cent.

The efficiency may also be found without reference to $E$ or $I$ by equation 15 ,

$f=\frac{s_{o}-s_{o}{ }^{\prime}}{s-s^{\prime}}=\frac{1.115+0.115}{1.36-0.035}=92.8$ per cent.

The zero power factor curve in this case is the $80^{\circ}$ wave, since current and voltage are in quadrature at all points on this curve, $\beta=80^{\circ}+10^{\circ}$.

If we wish to find the sending end voltage and charging current of the above line at no-load, we lay off the $A$ line from the origin $1 / T_{o}=0$, and read the value of $1 / T$ and $s$ at the upper end of the $A$ line, whence,

$$
\begin{aligned}
1 / T & =0.494 / 78.2^{\circ} \\
h & \stackrel{\bullet}{=} \frac{s}{\cos \phi}=\frac{0.16}{\cos 78.2^{\circ}}=0.784
\end{aligned}
$$

then by equation 11,

$$
\begin{aligned}
& e=e_{o} \sqrt{\frac{h t}{2}}=60,000 \sqrt{\frac{0.784}{2 \times 0.494}} \\
& I=\frac{E}{N T}=\frac{53,500 \times 0.494 / 78.2^{\circ}}{382.5 /-10^{\circ}} \\
& \therefore=69,500 \text { volts } \\
& \quad=69 / 88.2^{\circ} \text { amperes, charging current. }
\end{aligned}
$$




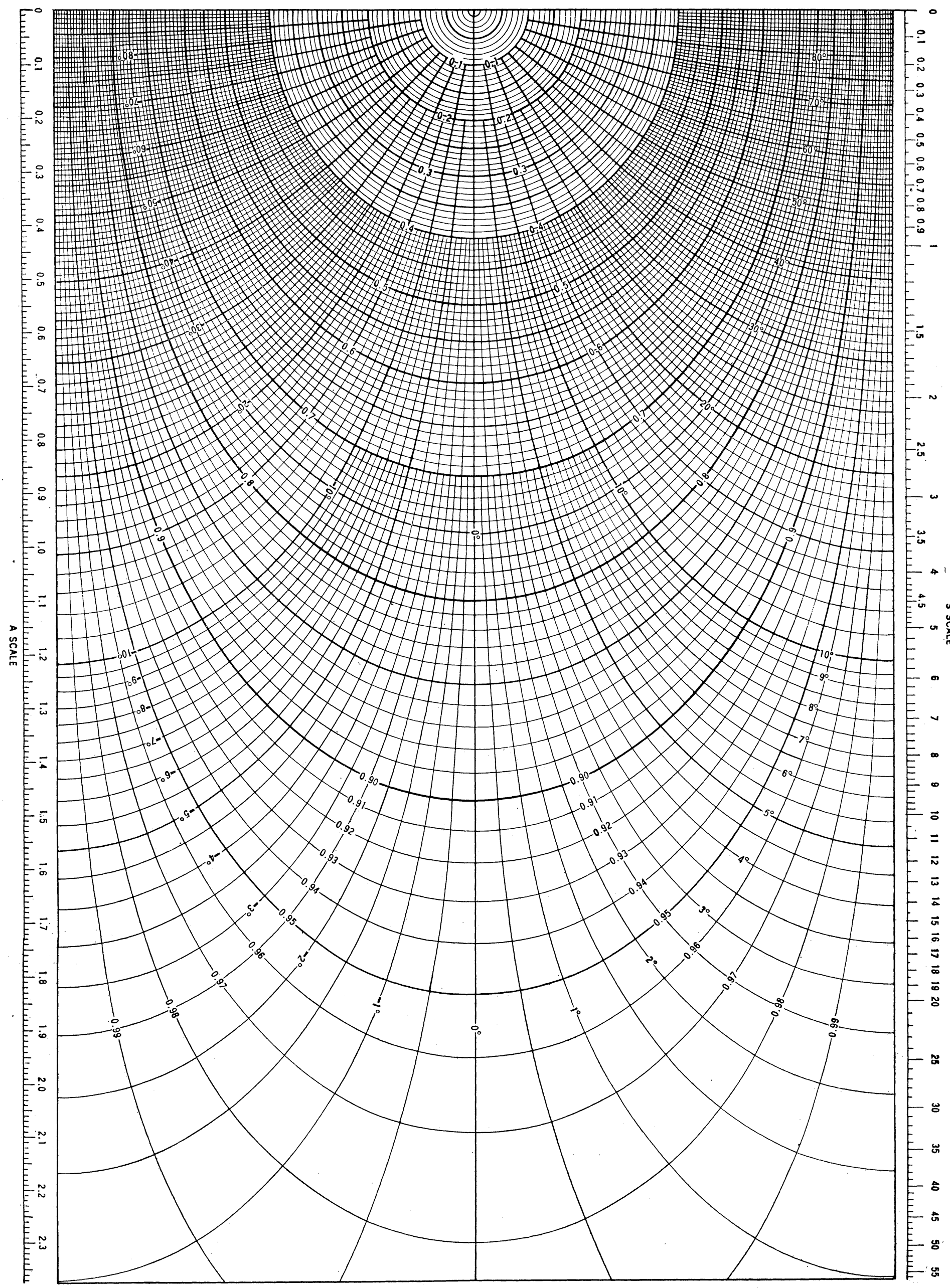

TANGENT CharT 
If we wish the sending end impedance with the distant end of the line grounded, $T_{o}=0$, the graphical part of the solution is the same as for the distant end free, with the result, $T=0.494 / 78.2^{\circ}$

$E / I=N T=382.5 /-10^{\circ} \times 0.494 / 78.2^{\circ}$

$=189 / 68.2^{\circ} \mathrm{ohms}$

\section{CONSTRUCTION OF THE WORK CHART}

Either one of two methods may be used in the construction of the work chart. First, the curves may be

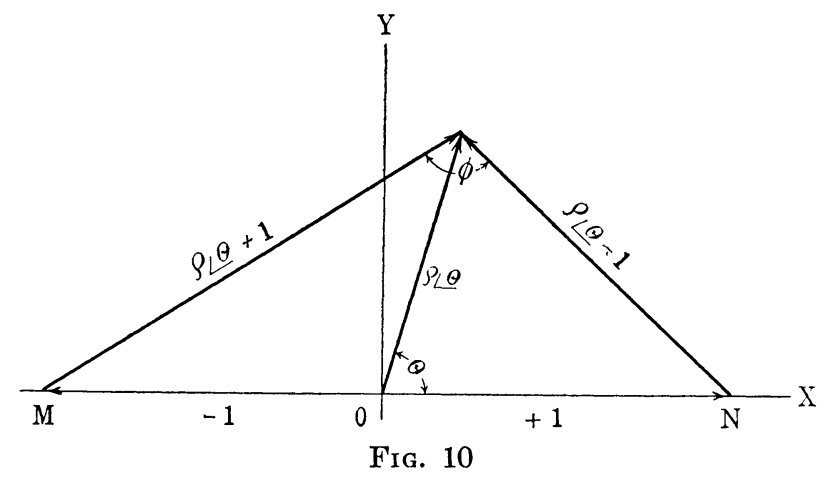

plotted from their scalar equations, $\mathbf{8}$ and $\mathbf{9}$, in the ordinary way, using tables of the hyperbolic functions and circular functions. Second, the curves may be plotted graphically, using an auxiliary diagram. This method does not require the use of either hyperbolic or circular functions. It is explained as follows.

$$
T=\tanh W=\frac{\sigma^{W}-\sigma^{-W}}{\sigma^{W}+\sigma^{-W}}=\frac{\sigma^{2 W}-1}{\sigma^{2 W}+1}
$$

Let $\sigma^{2 W}=\rho / \theta$, then,

$$
T=\frac{\rho / \theta-1}{\rho / \theta+1}=t / \phi
$$

A vector diagram of this equation is shown in Fig. 10. From it may be seen that if we construct a triangle with a base two units in length, the ratio of whose sides is $t$, and the angle at whose vertex is $\phi$, the line drawn from the midpoint of the base to the vertex will be the vector $\rho / \theta$. If $\phi$ is kept constant and $t$ varied, the terminus of $\rho$ will trace out a circle passing through $M$ and $N$ and having its center on $O Y$. If $t$ is kept constant and $\phi$ varied, $\rho$ will trace out another circle having its center on $O X$.

Here, then, we have another Argand diagram of orthogonal curves, Fig. 11, in which all the curves are circles and may be drawn with a compass. On such a diagram the $A$ line would appear as a logarithmic spiral.
The connecting link between the circle diagram and the tangent diagram is the relation,

$$
\rho / \theta=\sigma^{2 W}=\sigma^{2 u+2 j v}
$$

from which,

$$
\log \rho=2 u \quad \text { and } \quad \theta=2 v
$$

We may thus derive the tangent diagram from the circle diagram, which is relatively easy to construct. That part of the circle diagram which is needed to construct the top half of the tangent chart is included in the segment $A B N D$. This area converts into the rectangle of the chart.

After the circle diagram is drawn a scale of $2 u=\log \rho$ is accurately constructed by the aid of a table of logarithms. This scale is then laid on the circle diagram with its end at $O$, the value of $2 u$ read from the scale, and the corresponding value of $2 v$ read by means of a protractor. The resulting numbers, plotted in rectangular coordinates, yield the tangent chart.

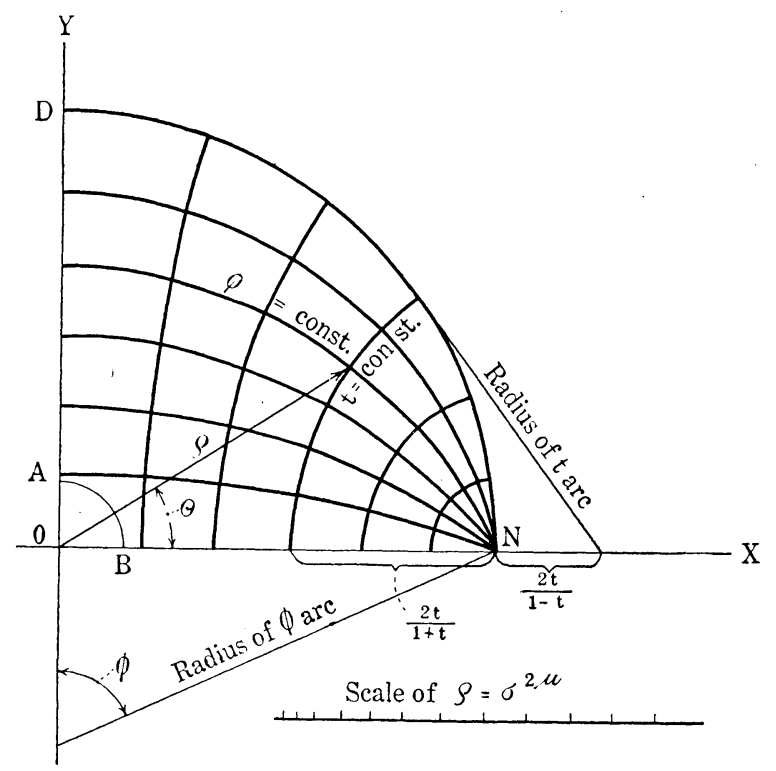

FIG. 11

\section{CONCLUSION}

It is hoped that the foregoing brief description of the tangent chart will interest others in this method with the result that means will be devised for using it in solving transmission line problems of even greater variety than those mentioned.

In conclusion the writer desires to express his sincere thanks to Messrs. Latour and Viard for their very excellent article on this method and to Dr. C. O. Mailloux for advice and suggestions in reference to the preparation of this paper. 